\title{
EL FRACASO ESCOLAR. ¿UN PROBLEMA DEL ALUMNO O DE LA INSTITUCIÓN?*
}

María Laura Maire

Adriana Yurkovich

(Alumnas de Ciencias de la Educación)

(Universidad Nacional de Rosario)

\section{Resumen}

Es la escuela la que fracasa. Los problemas de aprendizaje son problemas cognitivos. Un sujeto para aprender necesita: aparato psíquico constituido, aparato cognitivo, cuerpo y matriz de aprendizaje.

Hay tres formas de manifestar los problemas de aprendizaje:

- sintoma: que es el retorno de lo reprimido.

- inhibición cognitiva: que es la represión exitosa, es una disminución, es una evitación del contacto con el objeto de pensamiento, es un dispositivo puesto en marcha por el Yo frente a una situación de peligro.

- dificultades de aprendizaje reactivas: aquí es la institución socio educativa la que expulsa al aprendiente y promueve al repitente, no considerado como el que rehace un año, sino como el que repite lo que el docente quiere: el exitoso, es el que se acomoda al sistema, imita, repite, triunfa por repetir lo que otros quieren; y el fracasante, que provoca un problema al sistema que no lo acepta, que no reconoce su saber y lo obliga a acumular conocimientos.

* El siguiente Trabajo pertenece a alumnas de la Carrera de Ciencias de la Educación de la UNR (Extensión áulica Firmat). Ha sido avalado por las Prof. Adriana Usenky y la Prof. María Eugenia Annoni, integrantes del Aréa del Sujeto II.

\section{Palabras Clave:}

Fracaso escolar, Problema de aprendizaje, Aprendizaje, Constitución del sujeto, Pulsión epistemofilica 


\section{Summary}

It is the school the one that fails. The learning problems are cognitive problems.

A subject to learn needs: constituted psychic apparatus, cognitive apparatus, body and matrix of learning.

There are three forms to show the learning problems:

- Symptom: that it is the return of the repressed thing.

- Cognitive inhibition: that it is the successful repression, it is a diminution, it is an avoidance of the contact with the intention of thought, is a device started up by "YO" as opposed to a danger situation.

- Difficulties of reactive learning, are the educative institution here partner the one that it expels to the learner and it promotes to the repeater, not considered like that it remakes a year, but like which it repeats what the educational one wants: the successful one, is the one that complies to the system, imitates, repeats, prevails to repeat what others want; and the ones who fail, that causes a problem to the system that does not accept it, that does not recognize its knowledge and it forces it to accumulate knowledge.

\section{Key words}

Scholastic failure, Learning problem, Learning, Constitution of the subject, Epistemofílica pulsion

\section{Introducción}

El trabajo que presentamos consiste en un cruce bibliográfico de material trabajado en la cátedra de Sujeto II de la Carrera de Ciencias de la Educación de la Facultad de Humanidades y Artes de la UNR, en su Extensión Áulica de Firmat, y de otros textos que nos ayudan a esclarecer el tema seleccionado: FRACASO ESCOLAR.

Nuestro objeto de estudio es la categoria misma de "fracaso escolar", aunque sólo nos remitiremos a la comprensión teórica del mismo, es decir cuando éste se transforma en un problema; definiéndolo y diferenciándolo de categorias asociadas al mismo.

Plantearemos el "fracaso escolar" en su acepción más amplia, dejando de lado las causas deficitarias que requieren de una aproximación específica. Lo tomaremos desde la inhibición inconsciente del sujeto, inmerso en el contexto social, el entorno en general y otros factores que favorecen o traban el interés del niño por los asuntos escolares.
Para el desarrollo del trabajo se planea el análisis de categorías que atañen directamente al objeto de éste: aprender, deseo, sintoma, trastorno, problemas de aprendizaje, entre otros. $Y$ nos hemos planteado algunas preguntas para entender el tema que hemos escogido: ¿Qué es el "fracaso escolar"?, ¿Qué es aprender?, ¿Cómo se produce?, ¿Qué es un problema de aprendizaje?, ¿Cómo se construye el sujeto capaz de aprender?, ¿Qué papel juega la pulsión epistemofílica?

\section{Desarrollo}

"¿Es necesario definir el fracaso escolar?

Es una expresión que no deja a nadie indiferente, cada uno ajusta sus recuerdos felices o desventurados, en los que se mezclan arrepentimiento, nostalgia... a veces rencor. Existen aquellos a los que el fracaso escolar no les impidió triunfar en la vida y que se vanaglorian con eso, los que no se recuperaron jamás, y los que, felizmente más numerosos, no lo conocieron nunca." (1)

La categoría "fracaso escolar" es "de dudoso estatuto epistemológico, se inscribe en un campo incierto y problemático" (2). No obstante "...el discurso pedagógico instaura competencias cognitivas (saber hacer) y competencias pragmáticas (poder hacer), que interpelan al humano mediante modelos identificatorios con sujetos singularmente aptos $y$ poseedores de los conocimientos y condiciones necesarias -según el imaginario social- para acreditar un lugar en la complejidad de la organización laboral y el aparato productivo(Emmanuele, 1998:63)" (3), habilitándolo para ser entendido desde las hegemonías político- económicas y teóricas de turno.

Para comenzar a hablar del "fracaso escolar" es preciso recurrir a las ideas de Anny Cordiè (1994:18), con quien concuerda Alicia Fernández (2000:29) en la importancia de diagnóstico diferencial entre fracaso y problema. Según Cordiè el surgimiento del "término fracaso escolar es de reciente aparición, es una patología que aparece con la instauración de la escolaridad obligatoria a fines del siglo XIX" (4). "Cada época segrega sus patologías" (5).

Para Cordiè, el "fracaso escolar" es exclusivamente un sintoma que indica un malestar, sostiene que ninguna causa (sociedad, educación nacional, familiar, escuela, economia, etc.) puede explicar por si mismo el fracaso, ninguna generalización es válida para resolver o descifrar el tema. "Se considera que está en situación de fracaso escolar el niño que no

$$
-271-
$$


"sigue», porque en la escuela es necesario seguir: ante todo seguir el programa -que indica lo qué hay que aprender, en qué orden, en qué momento-, seguir a su clase, no alejarse del rebaño." (6)

Conviene aclarar que al hablar de sintoma, lo entendemos siguiendo las ideas de Fernández quien parte desde las conceptualizaciones de Laplanche $y$ dice:

...es un signo con significación simbólica... En el simbolo la relación significante-significado es menos arbitraria... Cada uno, a partir de su historia irá conformando estas relaciones.

El sintoma alude y elude al conflicto. Lo elude, para no conectarse con la angustia, pero al mismo tiempo esta mostrando una marca, señalando... aludiendo al conflicto. El síntoma es el retorno de lo reprimido. Es una transacción, que tiene que ver con una lucha entre instancias conscientes e inconscientes, para que aquello que se pretendió y se pretende reprimir se mantenga reprimido: no es algo que sucedió en el pasado y se sepultó. Hay una lucha constante... para que lo reprimido no aparezca. El retorno de lo reprimido aparece en forma transaccional y sustitutiva, a través de movimientos de condensación y desplazamiento... En todo síntoma... hay un mensaje encapsulado, el sujeto está hablando a través del síntoma con signos de un código poco o nada comunicable. Alude y elude. Denuncia y renuncia.

El código que elige el síntoma para hablar nunca se selecciona al azar. Si el síntoma consiste en no aprender, si el lugar elegido es el aprendizaje y lo atrapado la inteligencia, está indicando algo referido al saber u ocultar, al conocer, al mostrar o no mostrar, al apropiarse... el sintoma es como un disfraz. (7)

Los signos, considerados como una unidad psiquica de dos caras, significado y significante, son instrumentos subjetivos que al internalizarse autodirigen y regulan nuestra conducta y pensamiento. El hombre puede abstraerse, puede alucinar, puede pensar resolviendo un problema primero en su cabeza, usando signos ante la ausencia del objeto.

Pero a esta estructuración simbólica (8), a este referente subjetivo y dramático, donde inconsciente y Yo pujan por un "orden", hay que anexarle un plus, reconocido por todos los autores trabajados: el plus del contexto socio-cultural definido y delimitado en relación con otro.
Ya no podemos hablar de un sujeto epistémico o de un sujeto del deseo. Debemos hablar de un sujeto pedagógico (9) o sujeto autor (10), constituido en una relación entre enseñante, aprendiente y conocimiento inmersos en un contexto preciso y acotado en tiempo y espacio, donde cada uno de los elementos es indispensable para la conformación e identificación del sujeto, donde la transmisión y la transferencia son los hilos de esta red tejida con el uso de la lengua y del lenguaje. Lo orgánico, lo corporal, lo intelectual y lo simbólico (inconsciente), a los que hace referencia Fernández (1987: 63/75) se ponen en juego para poder aprender, son los bastidores de este telar.

Así, el hombre piensa. Por ello, nos resulta oportuno adherirnos a la propuesta de Sara Pain (1986: 16) "por definir una teoría única del pensamiento" que determine cómo se constituye el sujeto capaz de aprender, aunque para la dialéctica los conceptos no se definen, sino que se determinan.

El aprendizaje es una actividad especificamente humana que requiere del pensamiento, constituyéndose en una noción clave que articula inteligencia y sexualidad. Es el "proceso que permite la transmisión del conocimiento desde un Otro que sabe (un Otro del conocimiento) a un sujeto que va a llegar a ser sujeto, precisamente a través del aprendizaje." (11)

Es necesario aclarar que este "conocimiento del Otro no es solamente del Otro porque es Otro el que lo posee, sino porque en el aprendizaje este Otro es conocido o por lo menos reconocido como poseedor del saber." (12)

La transferencia de este saber, este proceso de transformación, se ubica según esta autora, entre dos polos: el primero, constituido por el que sabe, el enseñante, y el segundo constituido por el sujeto de conocimiento, el aprendiente, sujetado a una cultura peculiar, modalidad de construir la realidad humana.

Entonces tenemos por un lado al sujeto epistémico, del conocimiento, que construye sus estructuras cognitivas a través de la asimilación y la acomodación, que dan lugar a las competencias intelectuales que lo habjlitan para los aprendizajes. Un sujeto que tiende a adaptarse al medio. $Y$ por otro lado tenemos al sujeto deseante, que es un sujeto evanescente, dividido; su constitución es alienada en función de ese Otro y del lenguaje, que lo marcan de una manea y no de otra, distinta en cada sujeto.

Alicia Fernández logra unir estas dos categorias definiendo desde la psicopedagogia un sujeto propio, el sujeto enseñante-aprendiente, el sujeto de la autoria del pensamiento. 
Asi, sujeto aprendiente es pensado "como aquella articulación que van armando el sujeto cognoscente y el sujeto deseante sobre el organismo heredado, constituyendo un cuerpo, siempre en interacción con otro (conocimiento-cultura-...) y con otros (padres, maestros, medios de comunicación)." (13) Se construye a partir de dos posiciones subjetivas, simultáneas: la de Enseñante y la de Aprendiente.

Pero, ¿qué es el sujeto? Sujeto "que se escribe $S$, sujeto del inconsciente, marcado por la tachadura de la represión" (14), "barrado por el rechazo" (15), "... No es dueño absoluto ni de sus palabras ni de sus pulsiones. Esta carencia es estructural en él." (16)

¿A qué se debe esta falta? ¿Cómo se manifiesta?

La constitución del sujeto, como dijimos, es incompleta y deseante, es un ser-en-falta, como diría Raúl Ageno (1997) (17) y desde ahí surge el deseo y la búsqueda permanente por encontrar, en la respuesta del Otro, aquello que le falta para ser completo. $Y$ acá hablamos de la búsqueda de la certeza, ya que nuestro tema de trabajo nos compromete con el aprendizaje y por lo tanto nos ajustamos a ello

Para que un niño, un sujeto, "aprenda" es necesario que lo desee. Desde los primeros días de su vida un sujeto, se lanza a la exploración y al descubrimiento de su entorno y de su cuerpo. Lo habitan el deseo de saber, de comprender.

Este deseo de saber es lo que Freud equipara a una pulsión, la pulsión epistemofílica. Pero antes de hablar de esto es necesario que redondeemos un tema fundamental: la constitución del aparato psíquico.

Silvia Bleichmar (1995), desde la teoría psicoanalítica, propone una reconceptualización de la inteligencia y del pensamiento como una de las expresiones de la vida psíquica constituida desde Otro, que al mismo tiempo que sexualiza, inscribe al niño en la cultura en la que pensará, actuará y vivirá.

Es el pensamiento el que nos diferenciará de todas las otras especies, es la mediación dada por el otro humano, que nos parasita con sus sistemas (sexuales y representacionales), la que nos inscribe en la simbolización y la inteligencia. El inconsciente surge de esta adaptación y del proceso de constitución del sujeto como elemento de desadaptación. El inconsciente es el efecto de la fusión de las inscripciones (parasitismo simbólico o violencia primaria) (18) que el semejante instaura, sin saberlo, y por un estatuto, posterior, una represión que lo sepultará en los fondos del psiquismo, últimos recursos del aparato.
Si el otro humano se excede en estos actos, brindará un plus de excitación, que al no tener derivaciones obliga al aparato a una religazón, denominado por Freud (1895) fenómeno de la alucinación primitiva, donde los orígenes del pensamiento en lugar de relacionarse al objeto estarian vinculados a una alucinación

Como ya mencionamos hay una puja entre el inconsciente, que no tiene negación, temporalidad, significación como tal, y el Yo (Preconsciente) donde si están estas categorías, que se hace cargo de la autoconservación y de la producción de interrogantes. "La única posibilidad de conocer pasa por el yo, no pasa por el inconsciente. El inconsciente no puede más que reencontrar...aquello que tiene inscripto, por eso la alucinación se juega cuando ha caido la represión, cuando el inconsciente no ha sido obturado por el yo" (19).

Es el Yo el que toma las riendas del contacto entre la realidad psíquica y la externa, instaurando el triunfo de la civilización por sobre el instinto a través de la ley. Permite transformar los datos sensoriales, imponiéndole la barrera para resguardario de la realidad externa y al revés. "El aprendizaje reúne en un solo proceso la educación y el pensamiento, dando cumplimiento al principio de realidad, ...se basa siempre en una resignación..." (20) frente a la frustración de la barrera, que permite conformarse, de ser frustrado.

Retomando el planteo del deseo del saber, la duda, la curiosidad, nos lleva a la pulsión epistemofilica. "El niño transforma la curiosidad sexual previa, dirigiéndola hacia objetos de conocimiento socialmente aceptados. Esta derivación de la energía motiva el interés en la motivación... implica ...una represión exitosa y una derivación de la energía sexual." (21)

Para redondear, las actitudes del sujeto frente "...al objeto del deseo (de tener a la madre para si) y la ley (representada por el padre que prohibe la satisfacción del deseo), se definen según como se resuelva el conflicto edipico" (22). La ley lo somete, reprime su deseo y desarrolla distintas modalidades de relación. Ese deseo suspendido retorna de lo reprimido. Esta pulsión vinculada con el aprendizaje comienza con las preguntas: ¿De dónde vienen los niños?, ¿Por qué la diferencia de sexos?, ¿Qué es una relación sexual? Las represiones del adulto transforman la pulsión en una inhibición, un pensamiento compulsivo o una sublimación.

Otro aspecto en la estructuración del sujeto que hacen posible el aprendizaje son el narcisismo (modo particular de investidura libidinal), la configuración del ideal del yo (perfección e idealización del ldeal del Yo e identificación con los padres) y del Súper Yo (instancia critica que observa 
al yo y lo compara con el Ideal). "Las situaciones de aprendizaje implican poner en evidencia frente a los otros, la propia ignorancia, las fallas o carencias. El sujeto las puede negar ...o aceptarlas..." (23).

El niño realiza un pasaje del aprendizaje por el juego al aprendizaje que le concede la escuela, que es otra forma del saber. En este momento el proceso hace una pausa, hay un rechazo inconsciente a aprender, a entrar en este nuevo mundo, a este sistema de adquisición de conocimientos

Llegado este momento del trabajo consideramos necesario caracterizar al aprendizaje como:

..el proceso que sustenta la adquisición de conocimientos; se activa en el interior del ámbito escolar y puede ser descripto... desde una óptica exclusivamente subjetiva, pues no sólo representa el tipo de procesamiento que el niño hace de los conocimientos escolares, sino que constituye uno de los comportamientos a través de los cuales se expresan e interpretan aspectos importantes de la actividad psíquica individual. (24)

Sobre aprendizaje, R. Ageno dice "proceso (consciente e inconsciente) de adquisición de productos (conocimientos, habilidades, actitudes, valores, normas) que se obtienen al trabajar la materia prima que ofrece la realidad (informaciones, experiencias) con medios instrumentos adecuados (pensamiento, lenguaje, cuerpo), que reemplazan o resignifican anteriores adquisiciones." (25)

Por su parte Paín plantea: "el proceso de aprendizaje se inscribe en la dinámica de la transmisión de la cultura (...) si la continuidad del comportamiento animal está inscripta en su mayor parte en la disposición genética, la continuidad de la conducta humana se realiza a través del aprendizaje (...)" (26)

Consideramos que aprender es resignificar conocimientos anteriores: es entender la realidad de manera significativa, es un pasar de los conocimientos anteriores a nuevos complejamente a través de la incorporación y transformación de novedades que cada sujeto realiza al apropiarse de objetos, conocimientos. Como dice Pain es "un equivalente funcional del instinto" que lo diferencia de los de los animales, para así estar al tanto de la realidad e interpretarla. Es el aprendizaje el aval de la continuidad de la conducta humana. Solo como sujetos es que aprendemos, la estructura psíquica -cognitiva y deseante- accede a la elaboración, significación de los datos que la realidad le otorga. Lo central gira en que no se sabe todo; y esto hace que podamos posicionarnos como sujetos capaces de conocer o sea, investigar, errar, o poner en tela de juicio los conocimientos ya adquiridos. El pensamiento, cuerpo, lenguaje son indispensables herramientas. Es asi que el conocimiento al que accede el sujeto, es dado por otro, que es investido de un Saber. De alli que éste nunca es posible en soledad, es producido vinculando al que aprende y del que enseña.

En el aprender se articulan los siguientes elementos: Organismo individual heredado, Cuerpo constituido especularmente, Inteligencia autoconstruida interaccionalmente, deseo que es siempre deseo del deseo de Otro.

Según las ideas de Alicia Fernández (1987:91-92) los problemas de aprendizaje son de dos órdenes, por un lado, los que son internos a la estructura familiar e individual y se manifiestan como síntoma e inhibición (problema de aprendizaje-sintoma). Por otro, los externos a la estructura familiar e individual y que tienen que ver con la institución educativa, que rechace o desconozca la capacidad intelectual y lúdica, la corporeidad, la creatividad, el lenguaje y la libertad del aprendiente, por lo tanto el problema está en el saneamiento de la institución (problemas de aprendizaje reactivos). A estos últimos, Sara Pain, dice que son resultado de una sociedad «enferma - enfermante» que además de esto provoca oligotimia social (27).

La institución educativa es considerada como un lugar de articulación entre lo nuevo y lo viejo: entre los sujetos a que ingresan en un mundo que los precede. Es en este sentido que:

..las instituciones educativas, están ubicadas en el centro de un campo de tensión entre la tendencia a la conservación, a la reproducción, al más de lo mismo (forma ilusoria en la que se concibe al semejante) como requisito del lazo social y la tendencia a la innovación, la transformación, la revolución, la alteridad (o al reconocimiento del otro diferente) como requisito del lazo social. Es a partir de la problemática planteada por la alteridad que afirmaremos que la institución educativa es esencialmente intercultural (...) Por ello, y dado el carácter intermediario de la institución educativa, la cuestión cultural no puede permanecer como "el fondo nocturno de la actividad social', debe transformarse en figura. Vamos a sostener entonces que la interculturalidad es el contenido propio de la institución educativa, aquel sobre el que van a modelarse los conocimientos individuales. (28) 
Entonces Alicia Fernández (1987:98) plantea que hay tres formas de manifestaciones individuales de los problemas de aprendizaje: síntoma (retorno a lo reprimido), inhibición cognitiva (represión exitosa, no hay una transformación de la función, hay una disminución, una evitación de contacto con el objeto de pensamiento), dificultades de aprendizaje reactivas (la institución socioeducativa expulsa al aprendiente y promueve al repitente en dos vertientes: el exitoso, que es el que se acomoda al sistema, imita repite consignas, textos, no piensa, pero triunfa por repetir lo que otros quieren; el que repite de grado o fracasante: es el que provoca un problema al sistema que no lo acepta, que no reconoce su saber y lo obliga a acumular conocimientos)

Continuamos evocando a Alicia Fernández (1987:121 y s/s ) y ésta nos dice que, la modalidad de aprendizaje opera como una matriz que está en permanente reconstrucción y sobre la cual se van incluyendo los nuevos aprendizajes que van transformándola, pero la matriz es estructural. El sintoma cristaliza la modalidad de aprendizaje y a partir de ahí ésta pierde la posibilidad de ir transformándose y de utilizarse para transformar. El síntoma implica actuar lo que no se puede simbolizar, mientras que la simbolización permite resignificar y la resignificación posibilita que la modalidad pueda irse modificando. Cuando no se puede establecer este proceso de resignificación interno a la propia modalidad de aprendizaje, esta modalidad queda rigidizada, impidiendo o dificultando el aprendizaje de determinados aspectos de la realidad.

Así, Fernández, en otro texto (2000:29) distingue claramente 'fracaso escolar' de 'problemas de aprendizaje' que es imprescindible para poder intervenir antes de que se produzcan, ya que uno puede dar origen a otro.

"Entiende que el ser humano no es la sumatoria entre partes" (29) entonces "...el organismo transversalizado por el deseo y la inteligencia conforma una corporeidad, un cuerpo que aprende, goza, piensa, sufre o actúa." (30)

Ante este cuadro de situación podemos decir que "...en el «fracaso escolar», la modalidad de aprendizaje del sujeto no se patologiza, cuando se constituye un problema de aprendizaje (inhibición cognitiva o sintoma), la modalidad de aprendizaje se altera." (31)

Así en el "fracaso escolar" y en el problema de aprendizaje "el alumno muestra que no aprende, pero en el primer caso la patología esta instalada en las modalidades de enseñanza de la escuela, ya que ese es el lugar sobre el que prioritariamente debe intervenirse" (32).

Sabemos por nuestras prácticas que existen alumnos que padecen de un síndrome de fracaso o debilidad para el aprendizaje, los que dicen: "yo no puedo realizar el trabajo escolar porque soy torpe, no sé, no me sale". Estos alumnos atribuyen sus fracasos a la falta de capacidad más que a un esfuerzo insuficiente o al empleo de estrategias inadecuadas de aprendizaje. A menudo llegan a pensar que no pueden hacer ya nada para rendir satisfactoriamente, manifiestan actitudes derrotistas. La insistente comunicación de bajas expectativas por parte de los no-niños, adultos, a los que no se los puede llamar así ya que no han cumplido con la mayoria de edad de la que nos habla Kant; que rodean a los estos niños con dificultades con sus propias dificultades, y los otros niños, que los asedian, podría ser uno de los origenes de este modelo de esclarecimiento de los alumnos de bajo rendimiento.

Algunos docentes no suelen decirle directamente a los niños que tienen poca habilidad para aprender, pero lo sugieren: "Vos solamente contestá estas preguntas", "Hacé sólo este problema, o esta cuentita, o vos lee este cuentito", "Es suficiente con que leas del manual, esta hoja, esta parte". Otros docentes suelen no valoran sus pequeños éxitos o responden a los fracasos con sentimientos de compasión: "Pobrecito, no tiene libro de lectura", "Es una lástima que no le puedan comprar el manual", "Qué pena, ¡siempre pierde el cuaderno!", en vez de proponerles estrategias alternativas.

Determinados los docente suelen prestar menos atención a los alumnos de menor rendimiento, es más "lindo", "mejor" trabajar con los alumnos sin dificultades, con los que siempre responden, con los que traen el material para enriquecer la clase y hacer que la clase sea más atrapante. Generalmente los alumnos de menor rendimiento se sientan en el fondo, "porque no les interesa nada" o porque el docente le acerca un currículum menos rico en general, en objetivos, ejercicios, exigencias. Lo que agrava la situación del aprendiente.

Están también los docentes que sólo les piden que repitan las tareas: "Volvé a hacerlo más prolijo", "Volvé a leer varias veces esa lectura", como si la repetición fuese mágica y el refrán "la letra con sangre entra", una realidad.

Suelen criticar a esos alumnos por errores de aprendizaje que podrían no interpretarse como tales: "tenés faltas de ortografia por eso conviene que repitas muchas veces la palabra mal escrita". $\mathrm{O}$, por el contrario les preguntan con menos frecuencia o les plantean cuestiones más sencillas.

Mientras que los alumnos hacia quienes manifiestan expectativas positivas tienen más ocasiones de interacciones afectivas, más cálidas, se les

$$
-278-
$$

$$
-279-
$$


da más autonomía y responsabilidad para elegir y realizar los ejercicios. Como suelen decir los propios niños "son los preferidos".

Otro aspecto delicado de las bajas expectativas, es que el docente piense, con la mejor buena energía, que lo más conveniente es adecuarse a los ritmos propios de ellos, diferentes de los de la mayoría, sin advertir que de este modo, paradójicamente, rendirán cada vez menos.

Por último, también merece subrayarse que las expectativas de los docentes sesgadas de prejuicios "improntan" en el alumno y son sentidas por éste como mensajes discriminatorios o agresivos. Es natural que frente a ellas el alumno reaccione con conductas "molestas" que finalmente concretan los a priori negativos del docente.

Por otro lado es intrínseco de la educación la expectativa de futuro, su carácter prospectivo: formar al futuro ciudadano figura entre los fines de la educación de cualquier propuesta educativa, a pesar de que los docentes no siempre lo tengan presente.

Pero no es este aspecto al que queremos referirnos, sino a que el "adultocentrismo" en términos prospectivos es estructural en una escuela en la que predomine el modelo selectivo: "Este alumno va a llegar, va a poder seguir estudiando; en cambio, éste no".

¡Qué lamentable e imposible de evitar es esta rotulación! Esta biografía anticipada es bastante frecuente en la dinámica del ámbito educativo y constituye uno de los mecanismos sutiles de selección clasificatoria. Desde el ingreso a la institución, hasta el egreso o la expulsión; desde el apellido, la etnia, el género, la cultura o la religión.

\section{REFLEXIÓN FINAL}

"¿Por qué el éxito escolar ocupa un lugar tan grande en la vida de nuestros contemporáneos, niños, padres, educadores, gobernantes? ¿Qué proyectos, qué fantasmas recubren esta aspiración al éxito? (33)

El aprendizaje, cuando se torna un problema, pasa a llamarse "fracaso escolar". Esta temática es más abarcativa que el mero aprendizaje. Acá ya comenzamos a evaluar, a hacer estimaciones de lo que es el rendimiento promedio de un niño en torno a la perspectiva educativa y curricular que el medio demanda, en tiempo y saberes, muchas veces dejando de lado los procesos psicológicos que determinan sus producciones

De este modo en la historia "el llamado fracaso escolar se mantuvo durante décadas encapsulado en referencias estrictamente individuales" (34). Las figuras fueron la del inadaptado, la del retardado, la del desestructurado, la del disfuncional, la del disléxico, entre muchos otros; y sin olvidarnos de los estratos sociales. "el fracaso es visto como una de las consecuencias políticas económicas y sociales...Sin embargo...da cuenta siempre de resultados adversos para el sujeto portador del fraca- so; ya sea por carencias familiares, fallas pedagógicas o didácticas, o por obstáculos propios de la organización escolar." (35)

Al hablar de problema de aprendizaje, Fernández dice, "para poder entender su significado deberán descubrir la funcionalidad del sintoma dentro de la estructura familiar y acercarnos a la historia individual del sujeto y la observación de tales niveles operado. Para procurar la remisión de esta problemática debemos apelar a un tratamiento psicopedagógico clínico que apunte a desatrapar a la inteligencia y movilizar la patológica circulación del conocimiento en su grupo familiar" (36).

En este sentido, resulta interesante revisar la relación entre las expectativas del docente y el éxito o el fracaso escolar de los alumnos. Es importante tener en cuenta este análisis en la formación de los futuros profesores: cómo las expectativas de los maestros sobre el rendimiento de sus alumnos pueden convertirse en profecias que se cumplen por sí mismas, ya sea porque pueden aprender menos o más de lo que se espera de ellos.

Podemos asi definir al fracaso escolar "...como una problemática pedagógico-social que pertenece al ámbito institucional y que trata de dar cuenta del conjunto de factores que inciden o perturban la posibilidad de alcanzar niveles de conocimiento y de aprendizaje socialmente institucionalizados" (37).

La proyección de futuro no debe hacer perder de vista la situación presente, el aqui y ahora de la escuela, ya que es la escolaridad obligatoria la que se responsabiliza de garantizar educación para todos en el sentido del desarrollo de las potencialidades de cada uno, en la más amplia diversidad.

Concluimos que el peso social del fracaso escolar no puede medirse. No puede evaluarse.

Cientos de estadísticas y de números no marcan a diario los alumnos que abandonan el sistema, los que repiten, los que no ingresan. Pero eva- 
luar, medir, dar cuenta del fracaso escolar en si mismo es inmedible. Nadie mide su propio fracaso. Siempre la culpa es del profesor del año anterior. El que fracasa no es el alumno, es el sistema. El aprendiente solo tiene problemas de aprendizaje, más graves o menos graves; dificultades de aprendizaje en algún tema de una materia o en toda; tiene deseo de aprender o no.

Fracasa la escuela. Fracasa el sistema, el método, la formación, el contexto, la sociedad.

Tiene que ver con la pérdida de significación social de la escuela, donde uno de los factores que llevaron al quebranto de esta es la crisis de la función misma de la escuela, que es enseñar. La escuela no responde a la función social para la que fue instituida en su acto fundacional porque no enseña lo necesario para cubrir la demanda de aprendizajes básicos.

La bibliografia, aunque abundante y prolifica no logra poner en relieve las motivaciones inconscientes de cada sujeto, la resolución de las represiones, de la sexualidad. Freud nos dice que lo reprimido retorna en forma de síntoma y, si la escuela reprime las diferencias, el síntoma es el fracaso escolar, y va a ser distinto en cada sujeto, ya que cada uno es distinto al Otro.

Es así que, a lo podemos aspirar es a que el psicoanálisis y la psicopedagogía, junto con las buenas enseñanzas (38) y prácticas educativas logren liberar al sujeto de las exigencias de satisfacción sobre el mismo cuerpo y que pueda ubicar otro destino en el mundo.//I

\section{Notas Bibliográficas:}

(1) Cordiè, Anny. (1994) Los retrasados no existen. Psicoanálisis de niños con fracaso escolar. Ed. Nueva Visión Bs. As. Pp. 9.

(2) Annoni, María Eugenia y Caballero, Zulma. (2004)Fracaso Escolar: funcionalidad de un concepto esquivo en las intersecciones Clinica y Escuela- Colección Ensayos y Experiencias N55. Ediciones Novedades Educativas, Buenos Aires. Pp. 30.

(3) Annoni, M. Loc. Cit.

(4) Sevilla, Mónica. (2001) Temas Cruciales III. Fracaso Escolar. ED. Atuel-Infancia-Descartes. Bs. As. Pp.193.

(5) Cordiè, A. Óp. Cit. Pp. 15.

(6) Cordiè, A. Loc. Cit.

(7) Fernández, Alicia. (1987) La Inteligencia Atrapada. Abordaje psicopedagógico clinico del niño y su familia. ED. Nueva Visión. Bs. As. Pp. 95.
(8) Organizaciones cognoscentes que se realizan mediante la diferenciación de componentes objetivos, en una lógica de acciones, que se van construyendo para formar una representación. (N. de las A.)

(9) Concepto de Adriana Puiggrós (1990) en Imaginación y crisis de la educacićn latinoamericana, ED. Educación Alianza México DF. PP-41-42.

(10) Concepto de Alicia Fernández (2000) desde la psicopedagogia, en cuanto al sujeto aprendiente-enseñante en Los idiomas del aprendiente. ED. Nueva Visión. Bs. As. Pp. 58 y s/s.

(11) Fernández, A. (1987). Óp. Cit. Pp. 58

(12) Paín, Sara. (1986) La génesis del inconsciente. La función de la ignorancia II. Ed. Nueva Visión. Bs. As. Pp. 111.

(13) Fernández, Álicia. (2000) Los idiomas del aprendiente. Análisis de modalidades de enseñanza en familias, escuelas y medios. ED. Nueva Visión. Bs. As. Pp. 63.

(14) Cordiè, A. Óp. Cit. Pp. 31.

(15) Ibidem. Pp. 62.

(16) Ibidem. Pp. 177.

(17) Ageno, R. Colussi. G. (Comp.) (1997) El sujeto del aprendizaje en la institución escolar. Ed Homo Sapiens. Serie educación. Rosario.

(18) Castoriadis-Aulagnier, Piera (1993) La violencia de la interpretación. Amorrortu Editores. Bs. As. Pp. 33.

(19) Bleichmar, Silvia (1995) Aportes psicoanaliticos para la comprensión de la problemática cognitiva En Schlemenson, Silvia (Comp.) Cuando el aprendizaje es un problema. ED. Miño y Dávila, Buenos Aires. Pp. 28-29.

(20) Paín, Sara. (1973) Diagnóstico y tratamiento de los problemas de aprendizaje. Nueva visión. Cáp.2. Dimensiones del proceso de aprendizaje. Pp. 20.

(21) Fernández, A. (1987). Óp. Cit. Pp. 86.

(22) Ageno, Raúl. (1987) Psicología del aprendizaje. De El rol del psicólogo de la educación. En: Apuntes para una psicología de la educación. Cuadernos de Formación Docente $N^{\circ}$ 2- Publicaciones UNR. Pp. 17.

(23) Ageno, R. (1987) Óp. Cit. Pp. 18.

(24) Schlemenson, Silvia (Comp.) (1995) Cuando el aprendizaje es un problema. ED. Miño y Dávila, Buenos Aires. Pp. 12.

(25) Ageno, R. (1987) Óp. Cit. Pp. 7.

(26) Paín, Sara. (1973) Diagnóstico y tratamiento de los problemas de aprendizaje. Ed. Nueva visión. Cáp.1. Aprendizaje y educación. Pp. 9.

(27) Oligotimia social: capacidad de aprender y pensar, masivamente atrapada por deseosa de otros ordenes que no podriamos hacer corresponder con ninguna clase social en particular.

(28) Frigerio, Graciela. ¿Se han vuelto inutities las instituciones educativas? En Filmus, D. (Comp.) ¿Para qué sirve la escuela? Buenos Aires. Ed. Tesis Norma. 1994 pp. 134, 135, 137.

(29) Fernández, A. (1987) Pp. 63.

(30) Loc.cit.

(31) Fernández, A. (2000). Pp. 29 
(32) Ibidem. Pp.32

(33) Cordiè. A. óp. Cit. Pp. 28.

(34) Annoni, M.E. y Caballero, Z. Óp. cit. Pp. 31

(35) Fernández, Alicia. (1987) La Inteligencia Atrapada. ED. Nueva Visión. Bs. As Pp. 92.

(36) Schlemenson, S. Óp. Cit. Pp. 12.

(37) Schlemenson, S. Op. Cit. Pp. 12

(38) Buena enseñanza: Esta definición citada pertenece a Fenstermacher, en Fenstermacher, G. "Tres aspectos de la filosofia de la investigación en la enseñanza," en M. Wittrock La investigación en la enseñanza I. Barcelona: Paidós, 1989, quien considera que "el uso del adjetivo "buena" [enseñanza] no es simplemente un sinónimo de "con éxito", de modo que buena enseñanza quiera decir enseñanza que alcanza el éxito y viceversa. Por el contrario, en este contexto, la palabra "buena" tiene tanta fuerza moral como epistemológica. Preguntar qué es buena enseñanza en el sentido moral es preguntar qué acciones docentes pueden justificarse basándose en principios morales y son capaces de provocar acciones de principio por parte de los estudiantes. Preguntar qué es buena enseñanza en el sentido epistemológico es preguntar si lo que se enseña es racionalmente justificable y, en última instancia, digno de que el estudiante lo conozca, lo crea o lo entienda.(N. de las A)

\section{Referencias Bibliográficas:}

- Ageno, R. Colussi. G. (Comp.) (1997) El sujeto del aprendizaje en la institución escolar. Ed. Homo Sapiens. Serie educación. Rosario.

- Ageno, Raúl. (1987)El rol del psicólogo de la educación. En: Apuntes para una psicologia de la educación. Cuadernos de Formación Docente $N^{\circ} 2$ - Publicaciones UNR.

- Annoni, María Eugenia y Caballero, Zulma: Fracaso Escolar: funcionalidad de un concepto esquivo en las intersecciones Clínica y Escuela- Colección Ensayos y Experiencias N ${ }^{\circ} 55$. Pág. 28-38. Ediciones Novedades Educativas, Buenos Aires. 2004

- Bleichmar, Silvia. (1995) Aportes psicoanalíticos para la comprensión de la problemática cognitiva en: Schlemenson, Silvia (Comp.): Cuando el aprendizaje es un problema. Edit. Miño y Dávila, Buenos Aires.

- Castoriadis - Aulagnier, Piera. (1975) La violencia de la interpretación. Del pictograma al enunciado.Amorrortu Editores. Buenos Aires.

- Cordiè, Anny. Los retrasados no existen. Psicoanálisis de niños con fracaso escolar. Ed. Nueva Visión Bs. As. 1994.

- Fernández, Alicia.

a) (1993) La inteligencia atrapada. Ediciones Nueva Visión, Buenos Aires.

b) (2000) Los idiomas del aprendiente. ED. Nueva Visión. Bs. As.
- Paín, Sara

a) (1973) Aprendizaje y educación. En: Diagnóstico y tratamiento de los problemas de aprendizaje. Ed. Nueva visión.

b) (1986) El significado del aprender. En: Temas de Psicopedagogía $N^{\circ} 2$ Buenos Aires.

c) (1986) La génesis del inconsciente. La función de la ignorancia II. Ed. Nueva Visión. Bs. As.

- Schlemenson, Silvia (Comp.) (1995) Cuando el aprendizaje es un problema. Edit. Miño y Dávila, Buenos Aires.

- Sevilla, Mónica. (2001)Temas Cruciales III. Fracaso Escolar. ED. Atuel-InfanciaDescartes. Bs. As. 